\title{
Optical Biosensor Based on Microbendings Technique: An Optimized Mean to Measure the Bone Strength
}

\author{
Preeti Singh and Abhishek Shrivastava \\ University Institute of Engineering \& Technology, Panjab University, Sector 25, Chandigarh 160014, India \\ Correspondence should be addressed to Preeti Singh; preeti_singh@pu.ac.in
}

Received 3 September 2014; Accepted 9 December 2014; Published 24 December 2014

Academic Editor: Ralph Barry Johnson

Copyright ( 2014 P. Singh and A. Shrivastava. This is an open access article distributed under the Creative Commons Attribution License, which permits unrestricted use, distribution, and reproduction in any medium, provided the original work is properly cited.

Osteoporosis, a disease in humans, reduces bone mineral density. The microarchitecture of the bone gets deteriorated with change in variety of proteins in the bone. Therefore, a quantitative assessment of the strength of human bone, considering its structural properties and degradation due to aging, disease, and therapeutic treatment, becomes an integral part of the bioengineering studies. This paper presents a model of fiber optic biosensors (FOBs) which utilizes microbending technique to measure the strength of the bone. In parallel, an artificial neural network (ANN) based test bench has been developed for the optimization of FOBs strain measurement in orthoapplications using MATLAB. The performance accuracy of the given model appears to be considerable in ensuring the detection of the onset of osteoporosis.

\section{Introduction}

The importance of bone quality has long been recognized by orthopedic clinicians and radiographers to account for damage accumulation and predict susceptibility to fractures. There are numerous studies that have investigated the effects of age, drug treatments, and disease on the structure and properties of bone [1, 2]. Many of these studies employ biomechanical approach for testing stiffness, strength, and toughness of bone. Echo-tracking (ET) system $[3,4]$ has been mentioned in which strain of the bone is measured significantly under a certain load to evaluate the bone strength. But the given system needs further improvements, especially, to evaluate the measurement of displacement.

With the recent development of biosensors, it is now possible to monitor the strain on the bones. In this regard, various types of biosensors have been reported [5, 6]. Majority of them are based on piezoresistive strain gauge or other solid state sensing technologies. But their output is restricted to a small sensing area. Under the circumstances, it becomes necessary for the workers to use more sensors to sense larger regions. This is possible only at the expense of increased dimensions and loss of flexibility [7].

The limitations of conventional biosensors combined with poor compatibility of metallic components with human body, their larger sensitivity to EM interference, and also the fact that the majority of sensors guide electricity through metallic wires have restricted their application in clinical practice.

It is in this context that the FOS (fiber optic sensor) technology presents substantial advantages over conventional electric sensing systems. FOS is a device in which a low loss optical fiber is used in one or the other way. It can be used as a sensing element, also called intrinsic sensor. It can also be used as a means of relaying signals from remote sensor to the electronics that process the signals, well known as extrinsic sensor. FOS broadens the clinical application of sensors and also results in highly tested, mature, and precise measurements and biocompatibility.

FOS sensors guide the light and have many advantages. These are inertness, biocompatibility, low coefficient of thermal expansion and thermal conductivity, no electrical conductivity, immunity to EM interference, remote operation and sensing, small dimensions, being lightweight, adhesion to biological tissues, and geometrical versatility [7]. It makes FOS the tool of choice in the increasing number of sensing environments.

1.1. Optical Biosensors. In recent years, several investigations have been made regarding pressure biosensors and health 


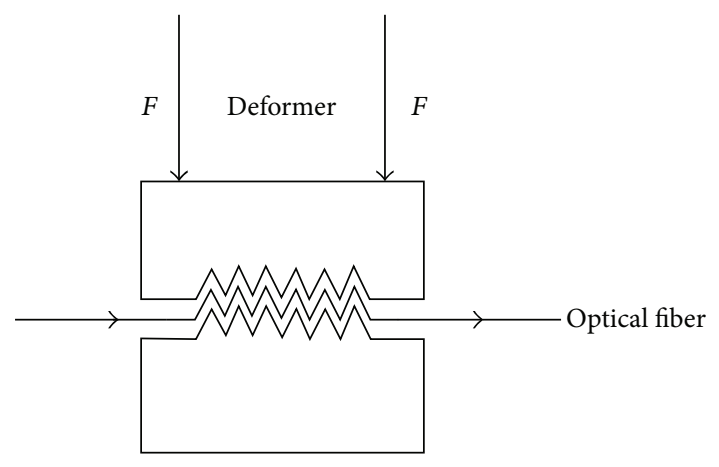

FIgURE 1: Microbending in optical fiber.

monitoring of structures. In this area, there has always been a requirement for developing a sensor that can be used as a time monitoring device in cyclic loading conditions for detecting the dangerous structural strain levels and also the failure of materials. An optical fiber implanted in a composite structure also gets deformed along with the structure itself. When there is a change in a physical parameter in the surrounding environment, the light passing through the fiber gets modulated. Using optical fiber sensing devices, the different physical and chemical parameters can be measured which describe the characteristics of light beams. These principal parameters include light intensity, phase, polarization, and wavelength. Among the various fiber optic sensing schemes, intensity modulation is the simplest and the cheapest way of detecting different parameters [8-12].

1.2. Intensity Modulated Microbend Sensors. In intensity modulated intrinsic FOS (as microbend sensor), the measurand modulates the intensity of the transmitted light through the fiber and these variations in intensity are measured using a detector situated at the output end of the fiber. Microbend sensors are based on excess transmission loss of a fiber to detect or measure the temperature, strain, pressure, displacement, and so forth. An optical fiber exhibits excess loss of light if a part of it is deformed. The optical fiber may be sandwiched between a pair of toothed plates to induce microbending. A phenomenon of microbending in optical fiber is shown in Figure 1.

The force $F$ is applied on the optical fiber sandwiched between a pair of toothed plates. It induces microbending on the optical fiber. The sharper the fiber bent, the more the number of waves lost at a particular radius of curvature as per fiber geometrics will be. The bend loss is extremely dependent on the radius of the bend and this fact is advantageous for microbend sensors. The wavelength (spatial) of periodic deformation is $\lambda_{s}$. When the phase matching state between the following couple of modes is satisfied:

$$
\beta_{p}-\beta_{q}=\frac{2 \pi}{\lambda_{s}}
$$

where, $\beta_{p}$ and $\beta_{q}$ represent the propagation constants (modal), then the transfer of power occurs from $p$ th to $q$ th mode. This power transfer will result in a total transmission loss of the guided modes, if $q$ th mode is a radiation mode. According to the mode coupling theory, for step index fiber $\lambda_{\mathrm{cr}}$, the critical wavelength (spatial) of the deformer is required to induce a significant transfer of power from the highest order guided modes to the radiation modes [13]. It can be expressed as

$$
\lambda_{\mathrm{cr}}=\frac{\pi \alpha}{\sqrt{\Delta}}=\left(\frac{\sqrt{\pi} \cdot a \cdot n_{1}}{\mathrm{NA}}\right),
$$

where $a$ is core radius of step index fiber, $n_{1}$ is refractive index of core, $\Delta$ is relative core cladding difference, $\alpha$ is profile index of fiber, and NA is numerical aperture of fiber.

Microbend induced loss in a multimode fiber is given by

$$
\propto=\frac{k a^{4}}{\Delta^{3} b^{6}}
$$

where $b$ is the radius of cladding and $k$ is the proportionality constant.

Such an optical fiber is proposed to be inserted inside the bone whose strain is to be measured.

A model of optical biosensor based on the microbending technique is designed to easily measure the strain on bones. In the present research work, multimode fiber is used.

\section{Proposed Design}

With the improvement in the area of biosensors to measure the strain on the bones, it is possible to more accurately detect the onset of osteoporosis. In the present paper, a model of fiber optic biosensor based on microbending technique is designed in the laboratory. The block diagram of the proposed model is shown in Figure 2 [14]. The accuracy of the designed sensor is checked with the aid of conventional strain gauge sensor. The hardware model of the biosensor designed is shown in Figure 3(a). On the basis of this model, a Simulink model is designed. It is shown in Figure 3(b). The strain versus attenuation plot is linearized using the data collected. The linear response of software model of biosensor design is shown in Figure 4 [15].

\section{Neural Networks}

In neural networks, different approaches are taken to solve a problem, different than the conventional intelligent machines, like computers. These networks process information in a way similar to a human brain. The neural network is comprised of a large number of highly interconnected processing elements called neurons. They work in parallel for solving a particular problem. There are input layer, hidden layers, and output layer consisting of neurons. In the present work, the concept of these neurons and layers is used to analyze the performance of the modeled biosensor.

\section{Proposed Approach}

The artificial neural network (ANN) based test bench has been developed for optimization of fiber optic biosensor for 


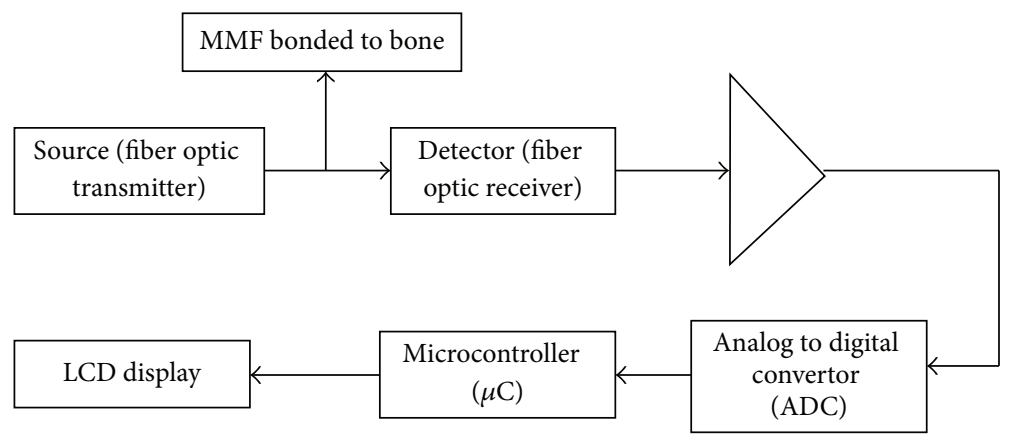

FIGURE 2: Block diagram of the proposed fiber optic biosensor [14].

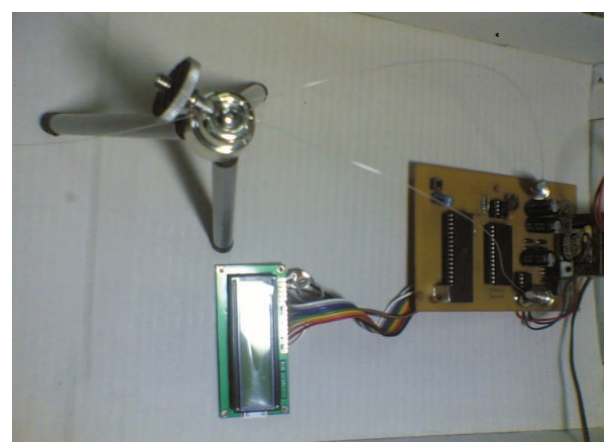

(a)

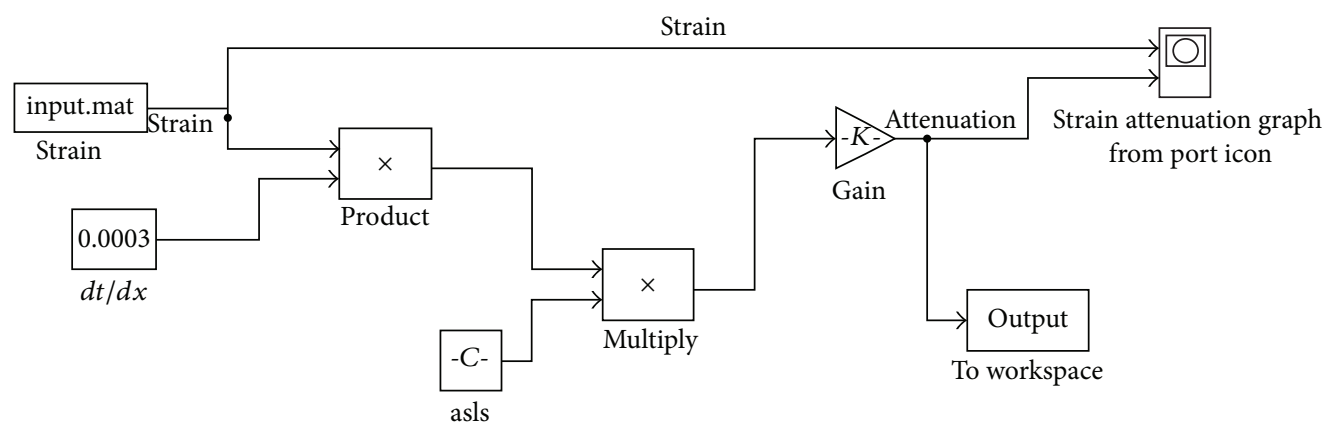

(b)

Figure 3: (a) Hardware model of biosensor. (b) Simulink model of biosensor.

strain measurement in orthoapplications using MATLAB. Subsequent iterations have been performed with ANN to analyze the performance of the Simulink model, first on the basis of number of layers and then with the number of neurons of the network.

\section{Results and Discussion}

In the present research work, ANN based biosensor is considered in terms of neurons and layers of a network.

5.1. Performance Analysis in Terms of Number of Layers. A series of simulations are carried out on the ANN based biosensor with one neuron. The percentage accuracy of the ANN based photometric strain biosensor trained using one neuron comes out to be $94 \%$ [14]. Now, the same neural network based biosensor is simulated for more numbers of neurons. The performance of the biosensor is analyzed in terms of mean square error (mse) versus the number of epochs for different values of neurons in the neural network.

The ANN simulation process consists of various sections like neural network system, algorithms, and progress and plots. A view of the neural network training tool with two neurons is shown in Figure 5. It can be observed from Figure 5 that the number of epochs is 25 when simulation with two neurons is carried out.

The number of epochs versus the training state parameters with two neurons is shown in Figure 6. The performance of neural network trained biosensor with two neurons in terms of mse is shown in Figure 7.

The graph in Figure 7 shows trends of trained, validation, and test data in terms of epochs versus mse. It is clear that, 


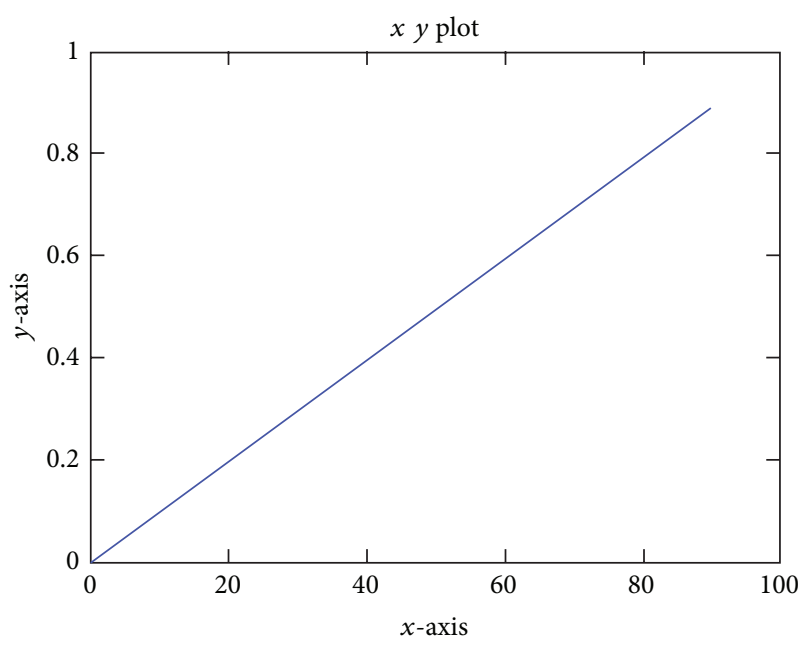

FIGURE 4: Response of model of biosensor ( $x$-axis: Strain; $y$-axis: Attenuation).

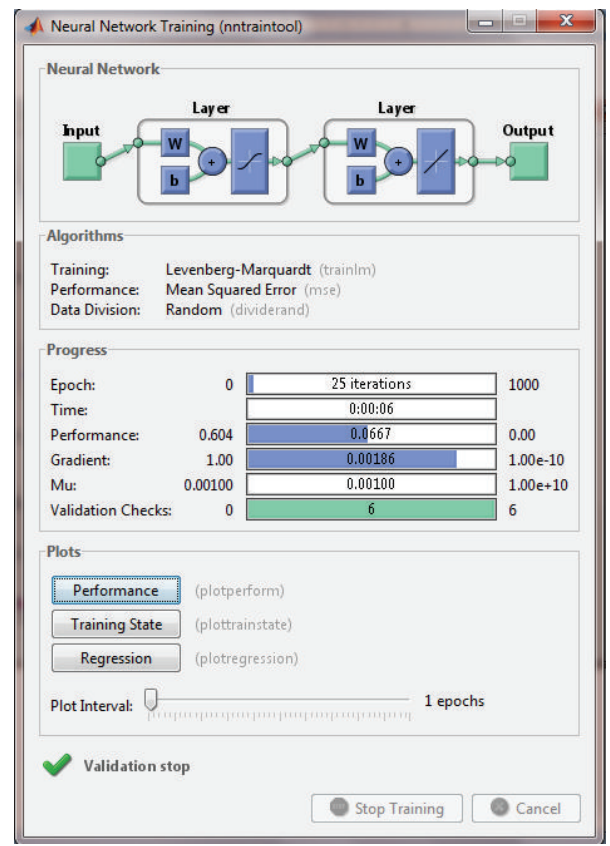

FIGURE 5: Neural network training tool showing simulation process for two neurons.

with the increase in number of epochs, the mse decreases for all trained, validation, and test data with slight difference in the slope. The best validation performance in terms of mse is 0.072079 with 19 epochs. On the basis of parametric performance, the percentage accuracy of designed biosensor for two neurons comes out to be $92 \%$.

It can be observed that, with the increase in number of neurons from one to two, the percentage accuracy falls from $94 \%$ to $92 \%$. Thus, there is a need to carry out simulations for more numbers of neurons for further analysis.

The performance of the biosensor is analyzed by increasing the number of neurons. The best validation performance in terms of the mean square error and the accuracy of the
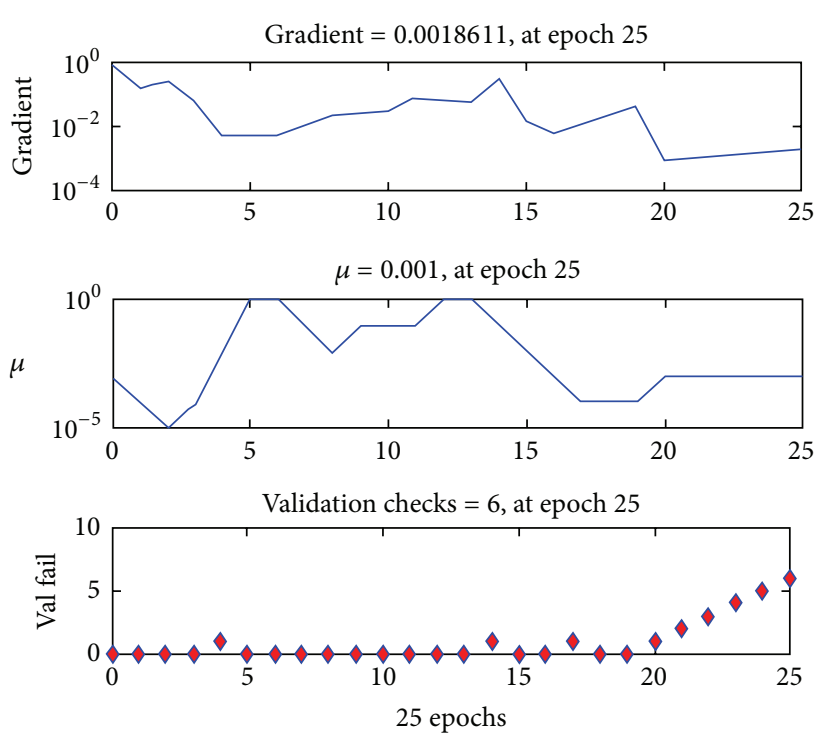

FIGURE 6: Number of epochs versus training state parameters with two neurons.

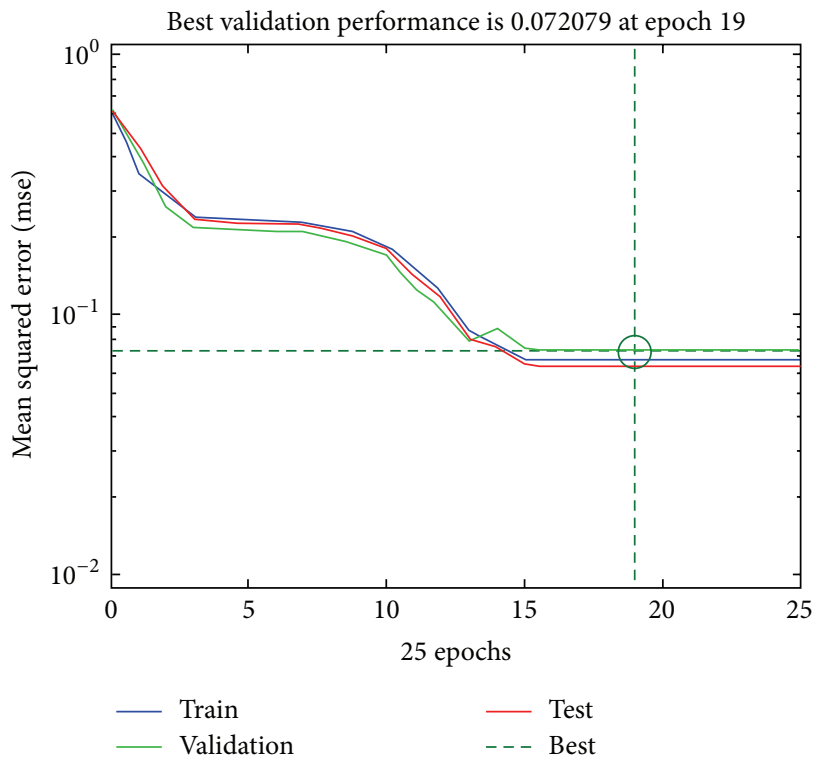

FIgURE 7: Performance of neural network trained biosensor with two neurons.

designed biosensor with six, eight, ten, and twelve neurons has been recorded and shown in Table 1. It is found that as the number of neurons is increased further, rise in percentage accuracy is observed.

The best validation performance with eleven neurons is observed in Figure 8 with mse as 0.033862 at 44 epochs. The optimized accuracy of the neural network based biosensor with eleven neurons comes out to be about $96 \%$.

When the simulation of the ANN based biosensor is carried out with twelve neurons, the best validation performance is observed in Figure 9 with mse as 0.039402 at 28 epochs.

The optimized accuracy of the neural network based biosensor with twelve neurons comes out to be about $96 \%$. 
TABLE 1: Performance analysis of neural network based biosensor with varying neurons.

\begin{tabular}{lcccc}
\hline $\begin{array}{l}\text { Serial } \\
\text { number }\end{array}$ & $\begin{array}{c}\text { Number of } \\
\text { neurons }\end{array}$ & $\begin{array}{c}\text { Mean square } \\
\text { error (mse) }\end{array}$ & $\begin{array}{c}\text { Percentage } \\
\text { accuracy }\end{array}$ & $\begin{array}{c}\text { Number of } \\
\text { epochs }\end{array}$ \\
\hline 1 & 1 & 0.044938 & $94 \%$ & 7 \\
2 & 2 & 0.072079 & $92 \%$ & 19 \\
3 & 4 & 0.059317 & $93 \%$ & 37 \\
4 & 6 & 0.037885 & $93 \%$ & 18 \\
5 & 8 & 0.030554 & $94 \%$ & 19 \\
6 & 10 & 0.031591 & $95 \%$ & 30 \\
7 & 11 & 0.033862 & $96 \%$ & 44 \\
8 & 12 & 0.039402 & $96 \%$ & 28 \\
\hline
\end{tabular}

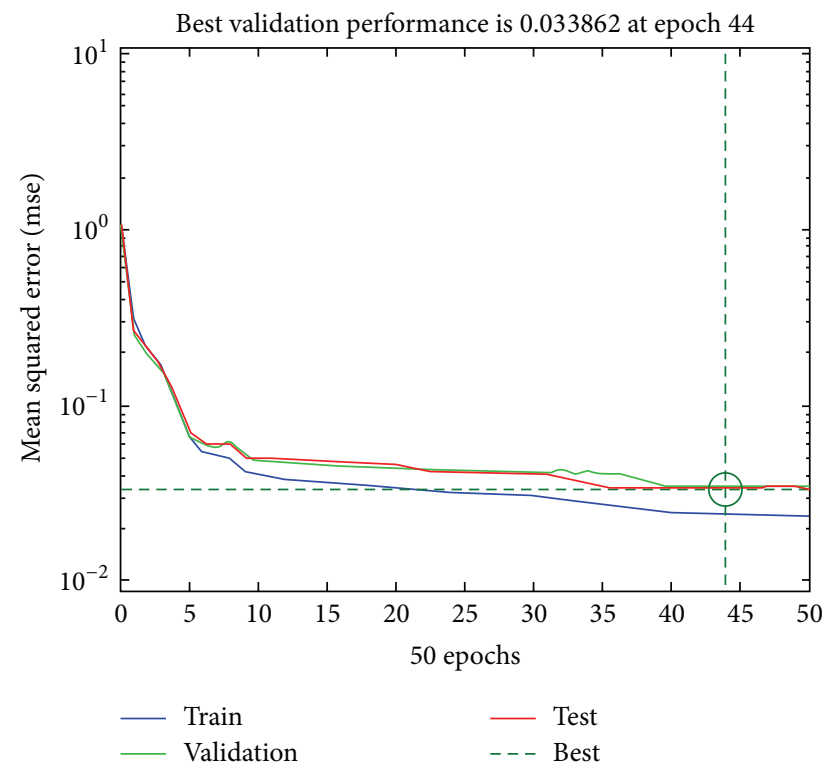

FIGURE 8: Performance of neural network trained biosensor with eleven neurons.

The performance of the designed system in terms of mean square error versus number of neurons has been analyzed. It has been observed that the outcome varies with number of neurons and, hence, the accuracy in percentage. The basic task here is reducing the mean square error between the actual output and the target output. As the value of mse decreases, the optimized accuracy of the ANN based biosensor increases for a given number of neurons. The training state parameters are standard for all neurons. The validation checks for all the simulations for varying neurons are six.

Figure 10 shows the variation in the result of the optimized percentage accuracy of the system designed with respect to the number of neurons. It shows that the percentage accuracy of the system is different for different neurons. The percentage optimized with one neuron is observed as $\approx 94 \%$ which decreases to $\approx 92 \%$ as the analysis is made for two neurons. In a series of simulations with a varying number of neurons, the maximum value of the percentage accuracy

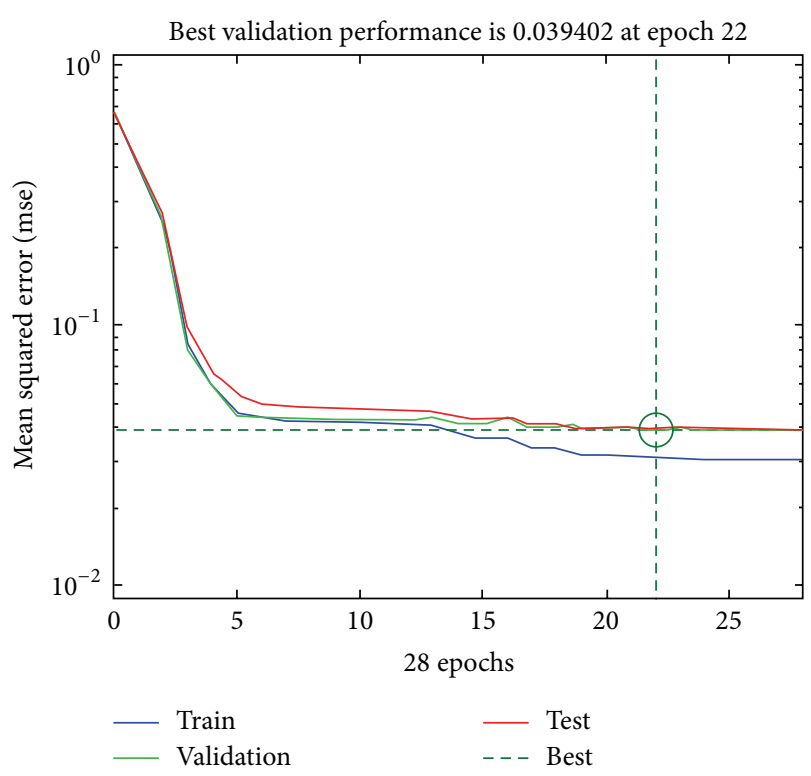

FIGURE 9: Performance of neural network trained biosensor with twelve neurons.

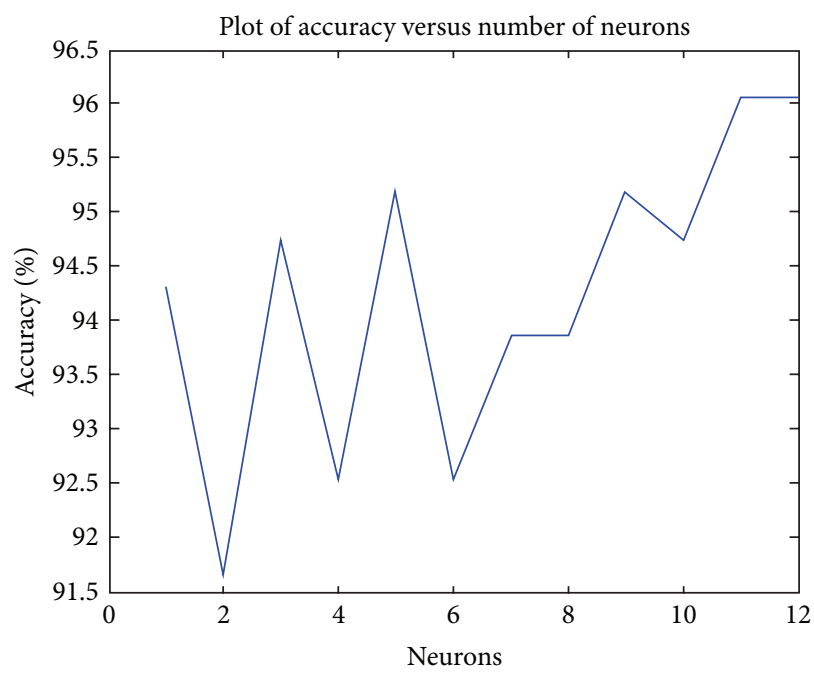

Figure 10: Accuracy versus number of neurons of neural network based biosensor.

comes out to be $96 \%$ and remains the same when number of neurons is eleven, twelve, or fourteen.

It can be seen that maximum accuracy of the designed system is achieved by using eleven neurons. Thus, the present neural network based biosensor is optimized to give maximum accuracy of $96 \%$ with eleven neurons. The plot in Figure 10 shows the variation of accuracy versus the number of neurons of the neural network based biosensor. It can be seen from the plot that maximum accuracy of the designed system is achieved by using eleven neurons.

The present work analyzes the performance of the fiber optic biosensor by varying the number of neurons. This work has been further extended to analyze the performance of the proposed sensor by varying the number of layers. 
TABLE 2: Performance analysis of neural network based biosensor with varying layers.

\begin{tabular}{lcccc}
\hline $\begin{array}{l}\text { Serial } \\
\text { number }\end{array}$ & $\begin{array}{c}\text { Number of } \\
\text { layers }\end{array}$ & $\begin{array}{c}\text { Mean square } \\
\text { error (mse) }\end{array}$ & $\begin{array}{c}\text { Percentage } \\
\text { accuracy }\end{array}$ & $\begin{array}{c}\text { Number of } \\
\text { epochs }\end{array}$ \\
\hline 1 & 1 & 0.043231 & $93.859649 \%$ & 27 \\
2 & 2 & 0.045623 & $92.543860 \%$ & 19 \\
3 & 4 & 0.049959 & $94.298246 \%$ & 6 \\
4 & 5 & 0.025603 & $96.929825 \%$ & 31 \\
5 & 6 & 0.035499 & $95.175439 \%$ & 28 \\
6 & 8 & 0.034076 & $93.421053 \%$ & 10 \\
7 & 10 & 0.029338 & $96.929825 \%$ & 10 \\
8 & 12 & 0.032027 & $95.614035 \%$ & 21 \\
\hline
\end{tabular}

5.2. Performance Analysis in Terms of Number of Layers. In this section, the performance analysis of the proposed biosensor is carried out with a varying number of layers. On the basis of parametric performance, the percentage accuracy of the system designed using ANN with one layer comes out to be $94 \%$ [14]. It has been observed that when the analysis with two layers is made, the percentage accuracy falls from $94 \%$ to $93 \%$. Thus, there is a need to carry out simulations for more numbers of layers. A series of simulations is carried out to analyze the performance of the biosensor by varying the number of layers from two to twelve.

The best validation performance in terms of the mean square error and the accuracy of the designed biosensor with different number of layers has been recorded and shown in Table 2. It is again observed from the table that, as the number of layers is increased, there is a rise in the accuracy of the percentage.

The best validation performance with five layers is observed in Figure 11 with mse as 0.025603 at 31 epochs. The optimized accuracy of the neural network based biosensor with five layers comes out to be about $97 \%$. In this way, the performance of the designed system in terms of mean square error versus number of layers has been analyzed.

Figure 12 shows the variation in result of the optimized percentage accuracy of the system designed with respect to the number of layers. It shows that the percentage accuracy of the system is different for different layers. The percentage optimized with one neuron is observed as $\approx 94 \%$ which decreases to $\approx 92 \%$ as the analysis is made for two layers. In the series of simulations with a varying number of layers, the maximum value of the percentage accuracy comes out to be $97 \%$, and it remains the same when number of layers is either ten or fourteen. The plot in Figure 12 shows the variation of accuracy versus the number of layers of the neural network based biosensor. Thus, the present neural network based biosensor is optimized to give maximum accuracy of $97 \%$ with five layers.

\section{Conclusion}

Strain measurement on bones has become easy with improvement in biosensors. Thus, it will be possible to more accurately detect the onset of osteoporosis. For such an

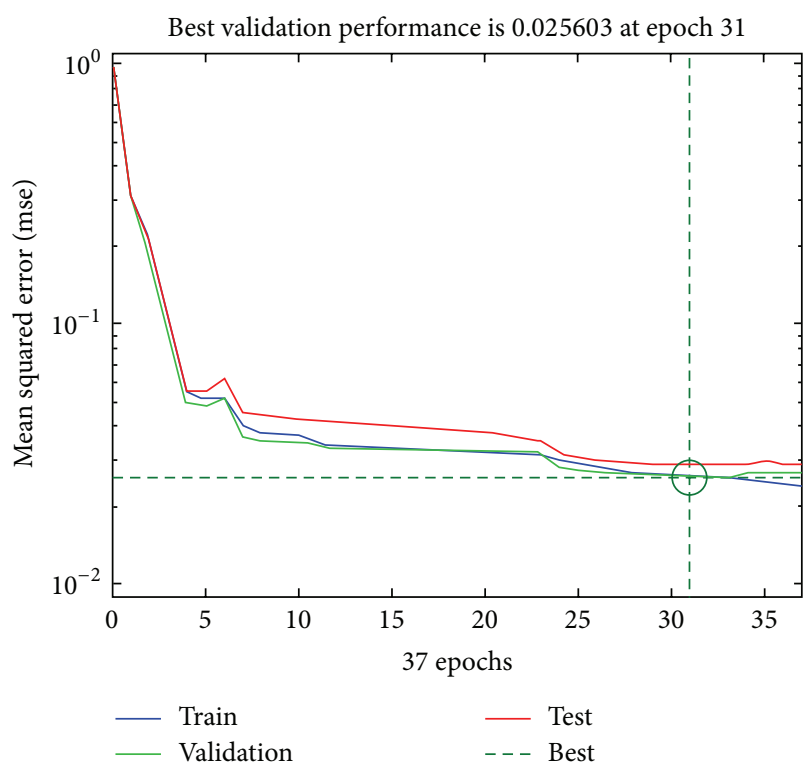

FIGURE 11: Performance of neural network trained biosensor with five layers.

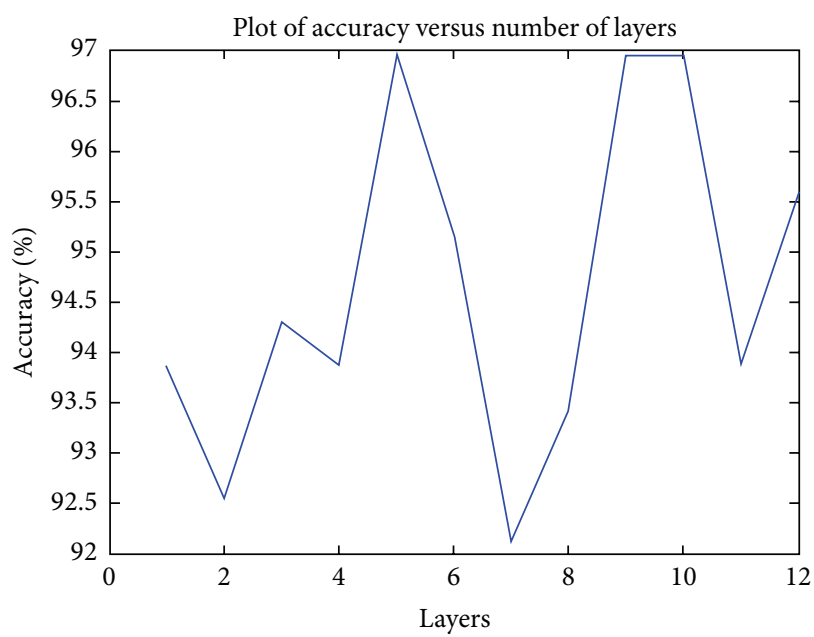

FIGURE 12: Accuracy versus number of layers of neural network based biosensor.

improvement, a model of photometric biosensor based on microbending technique is designed. The performance of the designed model is analyzed using artificial neural network by varying the number of neurons as well the number of layers. When the analysis with single neuron and single layer is carried out, the percentage accuracy is observed at $94 \%$ which gets reduced to $92 \%$ when the respective neurons and layers are increased to two. The performance of the designed biosensor is analyzed in terms of the mean square error. In the present paper, neural network based photometric biosensor based on microbending technique is optimized to give maximum accuracy of $96 \%$ with eleven neurons and $97 \%$ with five layers. The same level of accuracy has been achieved with an increased number of neurons and layers. But when maximum accuracy is obtained with less number, 
it is not worthy to design a complex system with an increased number of processing and structural elements. It gives better performance than what is available in the research literature.

The work carried out in the present paper is related to the fiber optic or photometric biosensors. It can also be continued to enhance the accuracy of other types of biosensors such as the potentiometric type or the potentiostatic type or to enhance the accuracy of biosensors such as those using discrete fiber optic sensors like Fabry-Perot interferometer or fiber Bragg grating, and so forth. Also, some other types of computation techniques such as fuzzy logic or particle swarm optimization can be used to further enhance the accuracy of the sensors.

\section{Conflict of Interests}

The authors declare that there is no conflict of interests regarding the publication of this paper.

\section{References}

[1] G. Balooch, W. Yao, J. W. Ager et al., "The aminobisphosphonate risedronate preserves localized mineral and material properties of bone in the presence of glucocorticoids," Arthritis and Rheumatism, vol. 56, no. 11, pp. 3726-3737, 2007.

[2] D. Taylor, J. G. Hazenberg, and T. C. Lee, "Living with cracks: damage and repair in human bone," Nature Materials, vol. 6, no. 4, pp. 263-268, 2007.

[3] A. Harada, R. Sakai, K. Miyasaka et al., "A new method for measuring bone strength using echo-tracking," in Proceedings of the IEEE Ultrasonics Symposium, pp. 3-16, 2006.

[4] R. Sakai, K. Miyasaka, H. Suzuki et al., "A minute bone bending angle measuring method using echo-tracking for assessment of bone strength," in Proceedings of the IEEE Ultrasonics Symposium (IUS '07), pp. 1116-1119, New York, NY, USA, October 2007.

[5] E. Ozcivici, S. Xu, A. Torab-Parhiz et al., "Cortical bone morphology and mechanosensitivity are modulated by genetic variations," in Proceedings of the 33rd Annual Northeast Bioengineering Conference, pp. 17-18, March 2007.

[6] T. Forster, C. Strohhofer, K. Bock et al., "Biosensor for calcium based on a hydrogel optical waveguide with integrated sensor proteins," in Proceedings of the International Solid-State Sensors, Actuators and Microsystems Conference, pp. 1218-1221, 2009.

[7] P. Roriz, O. Frazão, A. B. Lobo-Ribeiro, J. L. Santos, and J. A. Simões, "Review of fiber-optic pressure sensors for biomedical and biomechanical applications," Journal of Biomedical Optics, vol. 18, no. 5, Article ID 050903, 2013.

[8] L. Tang, Y.-M. Cha, H. Li, P.-S. Chen, and S.-F. Lin, "Fiber-optic immuno-biosensor for rapid and accurate detection of nerve growth factor in human blood," Proceedings of the 28th Annual International Conference of the IEEE Engineering in Medicine and Biology Society, vol. 1, pp. 811-814, 2006.

[9] M. Lee and P. M. Fauchet, "Label-free optical biosensor built with two-dimensional silicon photonic crystal microcavity," in Proceedings of the Conference on Lasers and Electro-Optics, pp. 1-2, 2007.

[10] S. Rahong, B. Saekow, S. Porntheerapat, J. Nukeaw, C. Hruanun, and A. Poyai, "Modification of the optical properties of polydimethylsiloxane (PDMS) for photonic crystal biosensor application," in Proceedings of the 3rd International Nanoelectronics Conference (INEC '10), pp. 1303-1304, Hong Kong, January 2010.
[11] D. Donlagic, Fiber Optic Sensors: An Introduction and Overview, Faculty of Electrical Engineering and Computer Science, University of Maribor, Maribor, Slovenia, 2000.

[12] N. K. Pandey and B. C. Yadav, "Fibre optic pressure sensor and monitoring of structural defects," Optica Applicata, vol. 37, no. 1-2, pp. 57-63, 2007.

[13] Preeti and H. M. Rai, "Modeling of fiber optic biosensor to measure strain on bones for detecting onset of osteoporosis," International Journal of Scientific and Engineering Research, vol. 3, pp. 1-3, 2012.

[14] P. Singh and H. M. Rai, "Performance analysis of photometric strain biosensor for bones using artificial neural network," International Journal of Computer Applications, vol. 54, no. 8, pp. 16-19, 2012.

[15] P. Singh, H. M. Rai, and Z. Singh, "Effect of neurons on the performance of microbend optical biosensor," International Journal of Engineering and Innovative Technology, vol. 1, pp. 233236, 2012. 

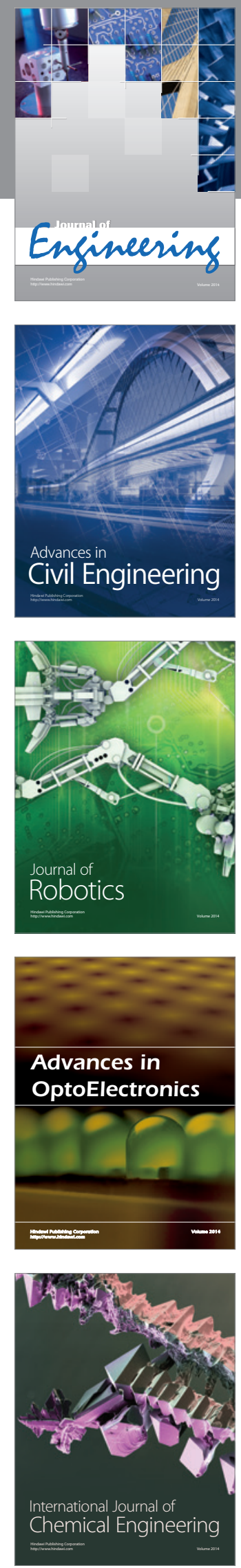

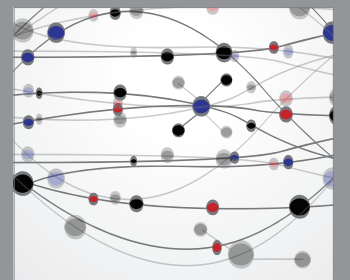

The Scientific World Journal
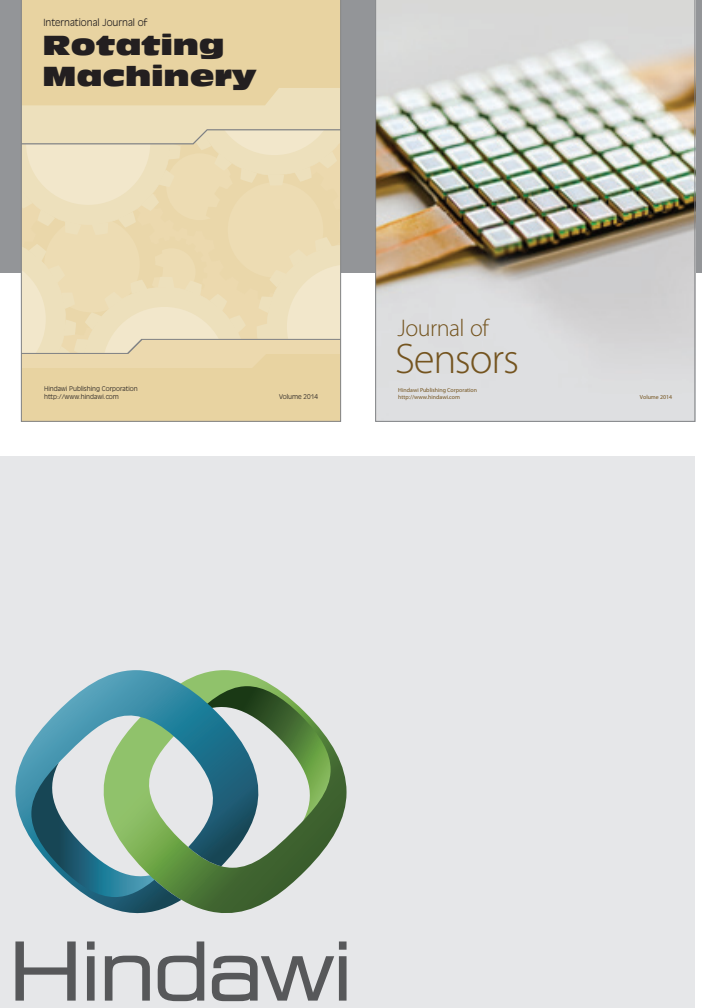

Submit your manuscripts at http://www.hindawi.com
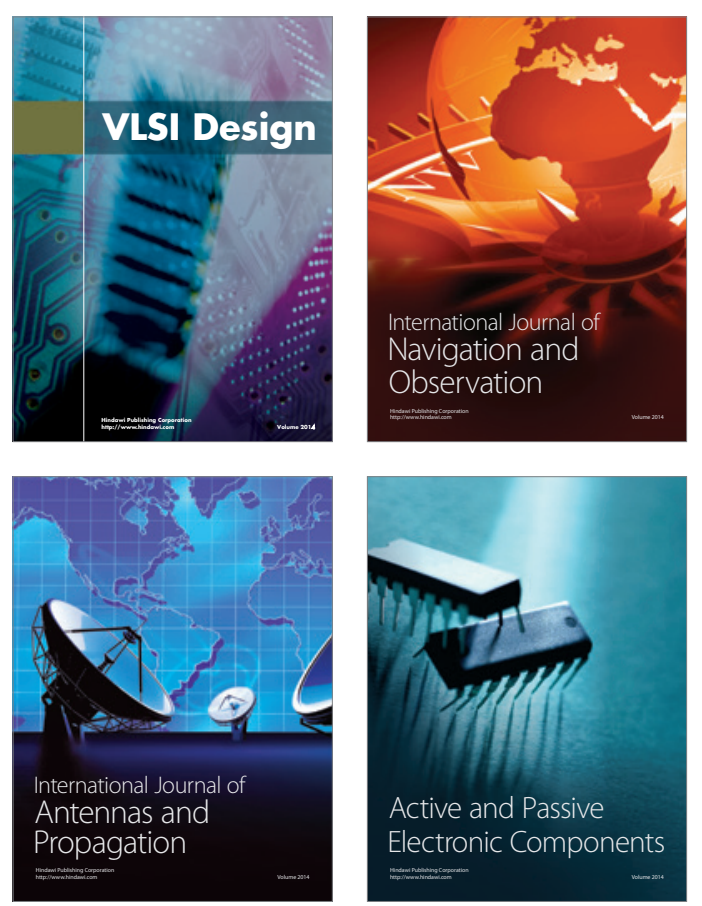
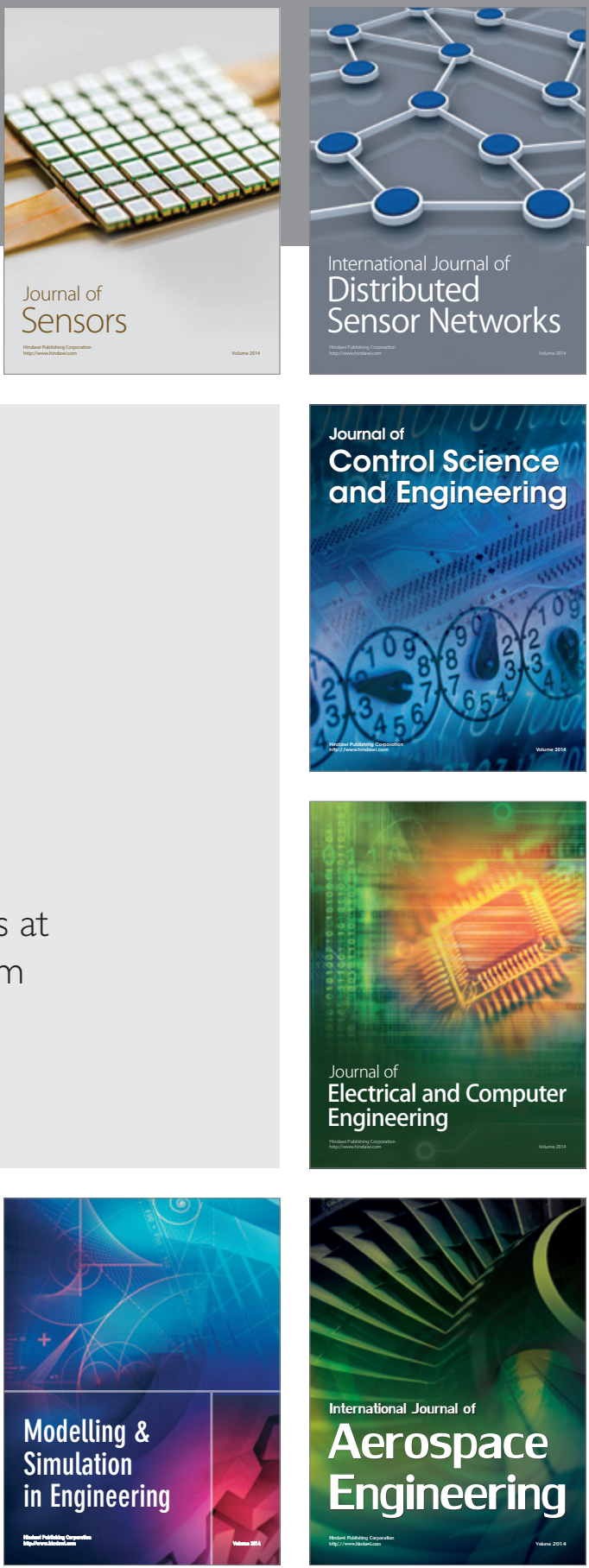

Journal of

Control Science

and Engineering
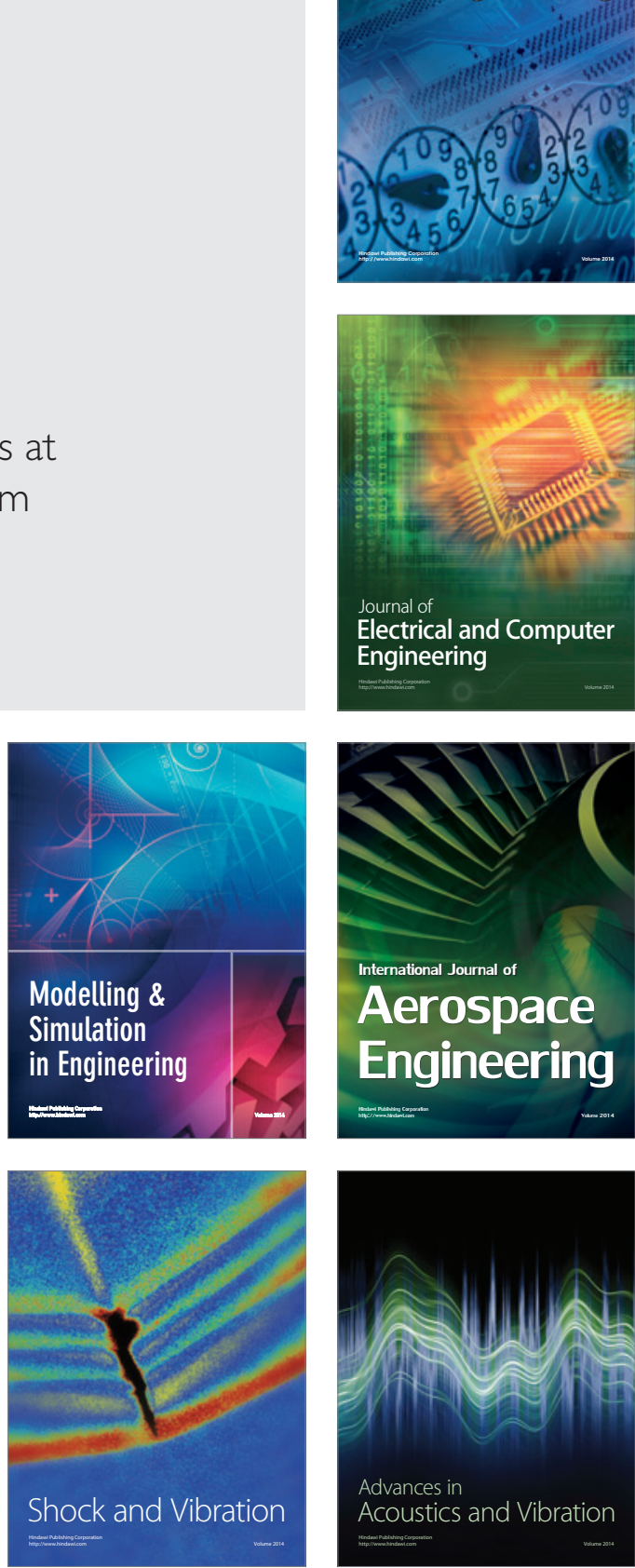